\title{
GALR1/GALR2 Knockdown rats block the Depression and Anxiogenic effects induced by GAL(1-15): The Heterodimer GALR1/GALR2 as a target of GAL(1-15).
}

\author{
C. Millón $^{1}{ }^{1}$, A. Flores-Burgess ${ }^{1}$, M. Narváez ${ }^{1}$, DO. Borroto-Escuela ${ }^{3}$, L. Santín ${ }^{2}$, C. Parrado ${ }^{1}$, J. A. \\ Narváez $^{1}$, K. Fuxe ${ }^{3}$, Z. Díaz-Cabiale ${ }^{1}$. \\ 1 . Faculty of Medicine, University of Málaga, Málaga \\ 2 . Faculty of Psychology, University of Málaga, Málaga \\ 3 . Department of Neuroscience, Karolinska Institute, Stockholm
}

The Galanin N-terminal fragment (1-15) [GAL(1-15)] induces depressant- and anxiogenic- like actions. In this work, we have studied the role of GALR2 and GALR1 on the effects of GAL(1-15) in the Forced Swimming Test (FST) and Open Field Test (OFT) using siRNA GALR2 and GALR1 knockdown rats.

Rats ( $\mathrm{n}=6-14)$ were injected with GAL(1-15) 3nmol, GALR2 antagonist M871 $3 \mathrm{nmol}$ in combination or alone 15 before the FST or OFT. The time of immobility, climbing and swimming were recorded during 5 min FST and Time and entries in the central square during $5 \mathrm{~min}$ were scored in the OFT.

In other experiment, rats $(\mathrm{n}=6-14)$ were injected Intracerebroventricular (icv) with siRNA-GALR2 or siRNA-GALR1 to generate the GALR knockdown rats. These knockdown rats were used in the OFT and in the FST after receiving icv GAL(1-15) $3 \mathrm{nmol} 15 \mathrm{~min}$ before the test. Vehicle was used as control.

In the FST, M871 significantly blocked the increased immobility $(\mathrm{p}<0.001)$ and decreased climbing $(\mathrm{p}<0.01)$ induced by GAL(1-15). In the OFT M871 also significantly decreased the number of entries $(p<0.001)$ and time spent in the center $(p<0.05)$ mediated by GAL $(1-15)$.

Down-regulation of GALR2 or GALR1 by siRNA was sufficient to block the effect of GAL(1-15) in behavioural tests. Thus, GAL(1-15) 3nmol lacked effect on the immobility, climbing and swimming time in the FST. The same effect was observed in the number of entries and time spent in the central square in the OFT.

These results indicated that GALR1 and GALR2 are involved in the GAL(1-15) depression- and anxiogenic-like effects suggesting that GAL(1-15) could act through GALR1/GALR2 heteroreceptor complex. These findings may give the basis for the development of novel therapeutic drugs targeting GAL(1-15) system for treatment of depression and anxiety disorders.

This study was supported by Junta de Andalucía CVI6476. 